\title{
Caracterización de las empresas procesadoras de productos lácteos de la ciudad de Estelí
}

\author{
Karolina Beatriz Moreno Moreno² \\ Arlen Meryfel Picado Juárez ${ }^{3}$ \\ Silvia María Rodríguez Solórzano ${ }^{4}$ \\ Beverly Castillo Herrera ${ }^{5}$
}

1 Este artículo se basa en la investigación titulada Caracterización de las empresas procesadoras de productos lácteos en la ciudad de Estelí en el año 2007. Para optar al título de Licenciatura en Administración de Empresas por la UNAN-Managua, FAREM-Estelí.

2 UNAN-Managua, FAREM-Estelí. Correo electrónico: kititol19@yahoo.com

3 UNAN-Managua, FAREM-Estelí. Correo electrónico: arlenmery@yahoo.com

4 UNAN-Managua, FAREM-Estelí. Correo electrónico: silviama2007@yahoo.com

5 Master en Ciencias Sociales por la Universidad de Guadalajara, estudiante del Doctorado en Ciencias Sociales de la Universidad del Zulia. Docente titular de la UNAN-Managua, FAREM-Estelí. Correo electrónico: beverly.

castillo@yahoo.com.

\section{RESUMEN}

Este artículo es resultado de una investigación que se realizó en 2007 sobre seis empresas procesadoras de productos lácteos instaladas en la ciudad de Estelí. Esta investigación permite conocer la problemática que enfrenta estas pequeñas empresas y sugiere una estrategia administrativa enfocada en la mejora de su producción y comercialización. Esta investigación es de aplicada y cualitativa, se describe y analiza el objeto de estudio de forma holística. Se utilizaron como estrategias de recolección de datos: entrevistas, encuestas, observación y grupo focal con empresarios del sector.

Palabras claves: Empresas lácteas, costo de producción, precio de venta, Comercialización, Calidad, Visión y estrategia empresarial. 


\title{
Characterization of the processing companies of dairy products of the city of Esteli ${ }^{1}$
}

\author{
Karolina Beatriz Moreno Moreno² \\ Arlen Meryfel Picado Juárez ${ }^{3}$ \\ Silvia María Rodríguez Solórzano ${ }^{4}$ \\ Beverly Castillo Herrera ${ }^{5}$
}

\begin{abstract}
1 This article is based on research entitled Characterization of dairy processing companies in the city of Esteli in 2007. To qualify for the Bachelor of Business Administration from UNAN-Managua, FAREM-Esteli

2 UNAN-Managua, FAREM-Estelí. E-mail: kititol19@yahoo.com

3 UNAN-Managua, FAREM-Estelí. E-mail: arlenmery@yahoo.com

4 UNAN-Managua, FAREM-Estelí. E-mail: silviama2007@yahoo.com

5 Master in Social Sciences from the University of Guadalajara, a PhD student in Social Sciences from the University of Zulia. Teaching holder from UNAN-Managua, FAREM-Estelí. E-mail: @ yahoo.com beverly. castillo.
\end{abstract}

\begin{abstract}
This article is the result of an investigation that was conducted in 2007 on over six dairy processing companies installed in the city of Estelí. This research provides information about the problems faced by these small businesses and suggests a management strategy focused on improving production and marketing. This research is applied and qualitative; the object of study is described and analyzed holistically. Interviews, surveys, observation and focus group with entrepreneurs of the sector, were used as data collection strategies.
\end{abstract}

Keywords: Dairy companies, production cost, sales price, marketing, quality, vision and business strategies. 


\section{INTRODUCCIÓN}

La ganadería ha sido históricamente una de las actividades económicas de mayor relevancia para los nicaragüenses, por ser uno de los medios más importantes de subsistencia para un amplio sector de la población rural del país. Según MSc. Ariel J. Cajina Loáisiga 120,000 productores dependen de la actividad ganadera bovina.

El Señor Alfredo Lacayo, Director Ejecutivo de la Empresa CENTROLAC, afirma: "A pesar de que tenemos una historia bien accidentada, siempre hemos sido competitivos en ganadería, siempre hemos producido mejor ganado al mejor precio y mejor leche al mejor precio que el resto de Centroamérica".

El hato ganadero nacional creció sostenidamente en los 60 y 70 pasando de 1.1 a 2.8 millones de cabezas. Se experimentó un descenso hasta 1.4 millones en la década de los años ochenta ${ }^{1}$. Sin embargo, la tecnología en este sector ha sido muy limitada, Ronald Blandón, Gerente General de la Comisión Nacional Ganadera, explica: "La ganadería nicaragüense está caracterizada por tener, generalmente, bajos niveles de incorporación tecnológica en sus sistemas de producción. En los años 60-70 esta actividad registró importantes avances en productividad, lo que fue impulsado por un repunte de los precios de la carne en el mercado internacional y por la existencia de un Banco de Desarrollo que dio financiamiento a corto, mediano y largo plazo". (Revista Envío. El Nuevo Diario. Lunes 20 de agosto de 2007).

Entre los años 1977-79, período que comprendió la lucha revolucionaria, muchas haciendas o fincas ganaderas fueron abandonadas porque en éstos lugares se daban enfrentamientos armados entre guerrilleros y la Guardia Nacional, provocando

1 Cajina Loaisiga, Ariel. La situación actual de la Ganadería Bovina de la Pequeña Agricultura en Nicaragua. Página 9 inseguridad, temor e incertidumbre entre los propietarios. Para tratar de reducir sus pérdidas, algunos productores decidieron llevarse gran parte de su ganado fuera del país. Sin embargo, muchos sufrieron robos, o se les murieron sus reses por falta de atención, esto provocó una disminución en el hato ganadero existente en Nicaragua.

El 29 de Febrero de 1980, durante la Revolución Popular Sandinista, se dio el decreto de expropiación de los bienes atendidos por el Instituto Nacional de Reforma Agraria (INRA), que afectaba principalmente a los productores relacionados con el Gobierno de Somoza. En las propiedades expropiadas por el gobierno sandinista organizó cooperativas y grandes empresas agropecuarias que conformaron el Área Propiedad del Pueblo (APP), el hato ganadero expropiado o abandonado fue incorporado a este tipo de actividad económica.

A inicios de los años noventa, la guerra había finalizado, pero la ganadería entró en el olvido, por parte de los gobiernos de turno, que hicieron casi desaparecer el financiamiento al sector agropecuario, aunque con el esfuerzo de los pequeños y medianos ganaderos, el rubro comenzó a levantarse hasta colocarse como uno de los rubros que más contribuyen a la economía nacional. Desde el 2004, en Nicaragua la producción de leche ha tendido a incrementarse, llegando a producirse unos 320 millones de litros anuales aproximadamente. Nicaragua ha pasado a ser un exportador neto de lácteos, en el 2003 se llegó a exportar sesenta millones de dólares.

Entre enero y julio del 2007 el país exportó leche, carne y ganado en pie por valor de 182.9 millones de dólares, 51.1 millones de dólares más con relación a este mismo período en 2006. La carne industrial es el producto pecuario que más se incrementó, pasando de 79 millones a 101.1 millones de dólares, seguido de los productos lácteos y del ganado en pie en tercer lugar (Envió-revista mensual de análisis de Nicaragua 
y Centro América. "ganadería nacional con buen futuro". El nuevo diario lunes 20 de agosto de 2007).

En los últimos 10 años la única fuente de financiamiento ha sido la banca privada pero, por los altos intereses, a corto plazo no es una solución para los ganaderos del país.

\section{La producción de leche en el municipio de Estelí}

En el municipio de Estelí, la producción de leche es altamente estacional, existiendo un período de concentración de la oferta que va de mayo a septiembre y comúnmente conocido como "Golpe de Leche".

En el municipio de Estelí han existido muy pocas empresas exportadoras de productos lácteos, tal es el caso de "Productos Lácteos Membell" y "Lácteos Las Segovias". Estas empresas procesadoras se encargaban de distribuir su producción a nivel local y exportaban parte de su producción a otros países centroamericanos. Actualmente, "Productos Lácteos Membell" ya no exporta, y la empresa "Lácteos Las Segovias" cerró sus operaciones.

Los dueños de la exportadora "Lácteos Las Segovias" fueron la señora Manuela Robles y Carlos Robles, esta empresa dejó de funcionar hace unos cuatro años (2003). Estaba ubicada en el Barrio El Rosario. Su actividad era netamente de exportación, no vendían en el mercado local, exportaban toda su producción a El Salvador. Posteriormente sólo se dedicaron al acopio y venta de leche ${ }^{2}$.

Esta investigación se enfoca en pequeñas empresas familiares del municipio de Estelí, dedicadas al procesamiento de la leche para elaborar diferentes tipos de productos: quesos, crema, cuajada, quesillo

2 Esta información fue suministrada por Sr. Manuel Mendoza. La entrevista se realizó en Estelí, el 20 de Noviembre del 2007. y yogurt. Por sus características son empresas pequeñas y artesanales, sólo un $25 \%$ de la producción nacional de leche se transforma industrialmente. La infraestructura de las empresas artesanales no está diseñada específicamente para el procesamiento de la leche, generalmente utilizan un espacio de la vivienda familiar que adecuan para llevar a cabo el proceso productivo.

El equipamiento de estas empresas consiste principalmente en una descremadora, cinchos 5 para elaborar el queso, mesas para procesar el producto, canoas y tinas. Generalmente, los propietarios/ as de estas empresas no realizan inversiones e innovaciones tecnológicas en maquinarias y equipos especializados para garantizar una mejor calidad, diversidad y presentación de los productos.

En este tipo de empresas trabajan un promedio de cinco personas, que se encargan de la elaboración, empaque y distribución del producto. La empresa les facilita a sus trabajadores batas, gorros, mascarilla, guantes y botas para que ellos lo utilicen al momento del procesamiento. El Ministerio de Salud (MINSA) se encarga de la salubridad en estas pequeñas procesadoras y dan los permisos sanitarios para que operen, pero se les exige cumplir con ciertos requerimientos, tales como: fumigación del local, certificados y exámenes de salud a los trabajadores, y supervisión del área de higiene y epidemiología.

Estas empresas procesadoras de Estelí tienen volúmenes de producción bajos, en comparación con industrias más desarrolladas como la Perfecta o la Parmalat, que tienen cobertura nacional. La mayoría de los productos que elaboran se comercializan directamente en el propio local, donde llegan los mayoristas que son compradores individuales que luego revenden el producto en pulperías, minisúper, restaurantes y comedores. También son proveedores de supermercados o instituciones como: la Policía, el Ejército y el Sistema Penitenciario "Puertas 
de la Esperanza", entre otros. En este caso, las procesadoras llevan directamente el producto al local del comprador. Algunas procesadoras tienen su propio medio de transporte, esto les permite distribuir sus productos a nivel local y unos pocos a otros municipios como: Condega, Somoto, Ocotal, Palacagüina, entre otros.

Actualmente, ninguna de las procesadoras de la cuidad de Estelí exporta sus productos porque requieren de altos volúmenes de producción, una infraestructura adecuada y maquinaria adecuada para tal propósito.

En esta investigación se considera como uno de los problemas centrales la poca visión de desarrollo empresarial o actitud de emprendimiento de los propietarios, lo que repercute en el poco riesgo que asumen para ampliar su capital de trabajo, que les permitiría aumentar sus utilidades. Otras limitantes son la falta de caminos de penetración y facilidades de energía eléctrica en las principales cuencas lecheras del país, la escasa infraestructura de acopio refrigerado para leche, el escaso nivel de transformación industrial que limita producir lácteos con calidades mínimas para destinar el producto a la exportación y la falta de políticas y planes de apoyo al desarrollo del sector.

\section{MATERIALES Y METODOS}

La presente investigación es cualitativa porque pretende describir y analizar el objeto de estudio como un todo y está orientada más a las cualidades que presenta el problema en cuestión. El tipo de muestreo es No Probabilístico, y de tipo causal porque no todo el universo tiene la misma probabilidad de ser seleccionado. Dentro de los criterios de selección de la muestra están: abarcar el universo de las empresas procesadoras de lácteos de la ciudad de Estelí, disposición de los empresarios/as propietarios a ser entrevistados, y el acceso a la información documental requerida.
Para el cumplimiento de los objetivos que se plantean en esta investigación, se hizo uso de diferentes técnicas de recolección de datos, tales como: la entrevista, grupo focal, la encuesta, observación e información documental.

La entrevista se aplicó a un total de nueve personas, todos propietarios de las PYME's dedicadas al procesamiento de los lácteos de la ciudad de Estelí, cuya información nos permitió conocer más de estas empresas procesadoras y sus propietarios. El grupo focal se llevó a cabo en abril de 2007 en la Biblioteca Urania Zelaya de la Facultad Regional Multidisciplinaria (FAREM-Estelí) con la participación de tres productores de las empresas lácteas de la ciudad de Estelí.

La encuesta se aplicó al azar a 30 consumidores de productos lácteos, el $70 \%$ eran del sexo femenino y un $30 \%$ del sexo masculino. Se realizaron directamente en los locales de la Ciudad de Estelí donde se comercializan los productos lácteos de las empresas estudiadas. Los locales fueron: Supermercado Las Segovias, Súper del Hogar, Minisúper Ray-Lun, El Pastoreo y El Quesito. Estos dos últimos locales son pequeños cafetines pertenecientes a las empresas procesadoras "Lácteos El Pastoreo" y "Lácteos El Quesito".

La observación se realizó en el área de producción de las empresas procesadoras para identificar las condiciones de los locales, las medidas de conservación y almacenamiento que utilizan, las Medidas higiénico sanitarias utilizadas por los empleados al momento del procesamiento

\section{RESULTADOS Y DISCUSION}

Caracterización de las pequeñas empresas procesadoras de lácteos en la ciudad de Estelí En este inciso se describe brevemente las características (propietarios, origen, capacidad y experiencia) de las 
seis empresas procesadoras de lácteos de la ciudad de Estelí estudiadas.

\section{Origen de las empresas procesadoras}

1. Productos lácteos el pastoreo: Esta empresa está ubicada frente al costado este del Instituto Guillermo Cano. Su propietario es el Señor Leonardo Pérez. Esta empresa nace en el año de 1993, con un grupo de productores que se afiliaron a una cooperativa, y así se empezó a procesar la leche que se producía en la finca de la familia Pérez y de los productores asociados. Tiempo después desapareció la sociedad y el Señor Pérez asumió la responsabilidad y propiedad de la procesadora.

Desde que se iniciaron operaciones hasta la fecha, se ha incrementado el volumen de producción en 10 veces más de lo que se producía antiguamente. Cuenta con una capacidad instalada de unos mil cuatrocientos litros por día. La empresa tiene entre 14 y 15 años de estar en funcionamiento.

2. Productos lácteos Mendoza: Productos Lácteos Mendoza está ubicado del Sopón tres cuadras al este, una cuadra al sur. Esta empresa inició sus operaciones en 1995, a partir de una idea del Señor Mendoza y un amigo suyo (un compadre) que trabajaba en una procesadora. Se empezó a trabajar en sociedad, la cual sólo duro tres meses, porque el socio no le vio futuro, ni utilidades y el Sr. Mendoza quedó trabajando sólo.

Se inició procesando 40 litros de leche, el proceso productivo se realizaba en la casa de habitación de la familia Mendoza. Pero, al incrementarse los niveles de producción se trasladó la planta a la casa de la suegra del Señor Mendoza, ubicada en el barrio José Benito Escobar. En la actualidad esta empresa procesa en un local destinado únicamente al procesamiento, este es un local propio, y se adquirió hace dos años. Se cuenta con una capacidad instalada de unos cuatro mil quinientos litros de leche diarios.

3. Productos lácteos el Quesito: Se encuentra ubicada de Ferretería Reynaldo Hernández ocho cuadras al este. Es una empresa familiar. La persona encargada de maneja la parte administrativa de esta pequeña empresa es Carmen Adriana Vílchez Castillo.

No se obtuvo la información suficiente para hacer la caracterización de esta empresa. Pero, según la guía de observación que se aplicó se puede afirmar que ésta es una de las empresas procesadoras más grandes de la ciudad, cuenta con su propia planta procesadora y con una capacidad instalada similar a la de "Productos Lácteos Mendoza".

4. Productos lácteos René Valle: Ubicada de Súper del Hogar tres cuadras al este. Sus propietarios Señor René Valle y su esposa la Señora Luz Mery Molina Moreno. Esta empresa tiene 20 años de funcionamiento. Comenzó con doscientos litros de leche, procedentes de la finca de la familia. Se contaba con una descremadora pequeña manual.

Inicialmente se procesaba en la finca, luego se trasladaron a Estelí para acopiar más leche y aumentar los volúmenes de producción, actualmente se procesa 1200 litros de leche diariamente en invierno es un poco más hasta 1500 litros diarios.

5. Productos lácteos "Eleazar briones": Ubicada de INISER dos cuadras y media al este. Se originó en el año de 1994. Esta empresa se crea como una alternativa de los padres del Señor Eleazar Briones Valle "Mi Papá trabajaba con ganado, la actividad comenzó como acopio y venta de leche al público en general; posteriormente se dio inicio al procesamiento de los diferentes productos donde vieron buenos rendimientos y ganancias". 
Se comenzó a procesar cien litros de leche, luego se incrementó a 400 litros y se llegó a trabajar hasta con 900 litros diarios. El padre del Señor Briones decidió cerrar operaciones. En 2002 decidió reabrir la lechería y comenzó procesando 80 litros de leche por día, luego 150 litros, y actualmente procesa entre 200 y 300 litros por día.

6. Productos lácteos "Miriam Rugama": Está ubicada del costado este de la Escuela Bertha Briones una cuadra al norte. Sus propietarios son la Señora Miriam Rugama y su esposo. Se inició operaciones hace 10 años, procesando 100 litros de leche y poco a poco se fue aumentando esta cantidad. Actualmente se está procesando una cantidad de 1,000 litros de leche diarios de lunes a domingo.

Ellos procesan en una casa cerca de su casa de habitación, donde habita otra familia. El empaque del producto es realizado por la Señora Rugama y su familia en su casa de habitación, en la cual cuentan con freezer para almacenar la producción.

\section{Tipo de productos que se elaboran}

Los productos que se elaboran en las pequeñas empresas procesadoras de lácteos de la Ciudad de Estelí son los siguientes: queso seco, queso seco ahumado, queso crema, queso con vegetales, queso mozzarella, queso parmesano, queso ricota, quesillo, cuajada, cuajada ahumada, cuajada ahumada con chile, crema y yogurt de sabores. Todos los productos lácteos son productos íntegros que se derivan del procesamiento de la leche. A continuación se especifican las características de cada uno de ellos:

1. Cuajada: A su componente principal que es la leche se le agrega cuajo líquido o en pastilla y un poco de sal. Su proceso es de una hora para obtener el producto terminado.

2. Cuajada Ahumada: El proceso es igual al de la cuajada normal, con la diferencia de que esta pasa por los hornos de ahumar para ser ahumada y luego se almacena porque es un producto seco.

3. Cuajada con Chile: Su proceso es igual al de la cuajada normal, el único componente adicional es el chile que le sirve como persevante y se le agrega un poco menos de sal que lo normal.

4. Queso Fresco: Es un producto tradicional procesado con cuajo y sal, todos los ingredientes se mezclan y el producto se coloca en prensas para desuerar y que este adopte la forma desea en el molde.

5. Queso Seco: Lleva el mismo procesamiento que el queso fresco, con la diferencia que se le agrega más sal de lo normal, ya que esta absorbe la humedad, luego se prensa en los moldes por dos días.

6. Queso Mozzarella: En este tipo de producto, la leche se descrema parcialmente, con sus mismos componentes. Luego, se desuera antes de ser colocado en una olla para fundirlo con el calor, se le agrega ácido cítrico y adquiere un aspecto de chicle, luego se coloca en moldes para que adopte la forma deseada.

7. Queso con Vegetales: Para su elaboración se realiza el mismo procedimiento del queso fresco, con la diferencia de que antes de ser prensado se le agregan pequeños trozos de chiltoma, cebolla, zanahoria y chile.

8. Queso Crema: Este tiene dos formas de elaboración, agregando crema y sin ella. La leche se pasteuriza a $63^{\circ}$, que es la temperatura más baja de pasteurización, y al alcanzar esta temperatura se baja a $45^{\circ}$ colocando el recipiente de la leche sobre agua, se agrega el cuajo y sal. Luego se hecha en moldes para ser prensados. Estos moldes tienen orificios por donde, poco a poco, el suero se escurre.

9. Queso Ahumado: Su proceso es igual al del queso normal, la diferencia es que pasa por un proceso de ahumado en los hornos y luego se almacena. 
10. Crema: Su procesamiento se realiza en la descremadora y sin introducir el producto a refrigeración para no matar las bacterias buenas que contiene la leche y que hacen que el producto se espese. La leche se coloca en un recipiente a temperatura normal o natural diaria, no se le agrega sal y su proceso para tener el producto terminado es de un día completo para luego ser empacada y vendida.

11. Quesillo: Lleva el mismo procesamiento que el Queso Mozzarella, con la variación de que se agrega menos cantidad de cuajo y se le puede adicionar suero ácido (añejo de varios días). Cuando el producto está terminado se le puede dar la forma de tortilla o de trenza.

Los productos más comunes como: la crema, cuajada y queso corrientes, son los que se producen en mayores volúmenes por el alto volumen de demanda que representan. Los otros productos como: queso con vegetales, queso ricota, queso crema, etc. no se producen en grandes cantidades, ya que no son muy comerciales y además su precio es más alto que el precio de los productos más comunes.

Casi en su totalidad las empresas procesadoras de lácteos existentes en la ciudad de Estelí se dedican al procesamiento de queso corriente, crema y cuajada; la única empresa que cuenta con una gama de productos más diversificada es Productos Lácteos "El Pastoreo" que además de los productos convencionales también elaboran diferentes tipos de quesos, quesillos y yogurt.

\section{Organización: capacidad instalada y maquinaria}

Todas las empresas procesadoras de la ciudad de Estelí son privadas y de carácter familiar. Cuentan con un personal aproximado de cinco personas por establecimiento. Estos se encargan de la adquisición de la materia prima, transporte, procesamiento, empaque y distribución del producto y administración de las empresas.
Estas empresas cuentan con la siguiente maquinaria: Tinas térmicas, descremadoras, cinchos, licuadoras industriales, freezer, termos, selladoras, termómetros, lactodensímetro, barriles plásticos, pichingas de plástico y aluminio, máquina de moler cuajada, máquina empacadora industrial y medios de transporte. Con esta maquinaria son capaces de procesar actualmente una cantidad promedio de 1,300 a 1,400 litros de leche diario, de lunes a domingo. El equipo que utiliza el personal para la elaboración del producto son: gorros, botas, guantes y gabachas. Ninguna de estas empresas está produciendo a su máxima capacidad actualmente.

\section{Los canales de distribución}

Los canales de distribución utilizados son:

- Productor-Consumidor Final: por que venden directamente a las personas que van a consumir el producto. Un ejemplo de este caso es Productos Lácteo René Valle que vende a la Policía y al Sistema Penitenciario.

- Productor-Intermediario-Consumidor: es el caso de las empresas que venden sus productos a intermediarios como: supermercados y pulperías, los cuales a su vez se encargan de hacerlos llegar al consumidor final.

La mayor parte de la producción es distribuida en la zona norte del país, en los municipios de: Somoto, Ocotal, Condega, Palacagüina, San Juan de Rio Coco y en la ciudad de Estelí. Además, se abastece a las ciudades como: Sébaco, San Isidro, Tipitapa, Managua, Granada y Chinandega.

Los locales que adquieren estos productos son: pulperías, supermercados, sistema penitenciario, pizzerías, policía nacional, el ejército, cafetines, distribuidoras, restaurantes y compradores al detalle. 


\section{La calidad de los productos lácteos procesados}

Existen ciertas fluctuaciones en el rendimiento debido a la baja calidad de la materia prima que se adquiere. Al momento del procesamiento no se da el rendimiento esperado ya que la leche no cuenta con la concentración necesaria de propiedades, vitaminas y minerales. Esto se da debido al cuido que recibe el ganado y también a las técnicas de ordeño utilizadas. En su mayoría, las personas encargadas de ordeñar el ganado usan medidas poco higiénicas y en algunas ocasiones mezclan el producto original (la leche) con cierta cantidad de agua.

Según un análisis físico químico realizado a la leche por el equipo multidisciplinario de FAREM, Estelí en la investigación "Diagnóstico de la Calidad y Comercialización de Leche y sus Derivados en el Municipio de Estelí" se encontraron los siguientes parámetros: ácido láctico $(0.190 \%)$, grasa $(3.86 \%$ ), densidad (1.032), pH (6.71) y alcohol(-). Los resultados están dentro del límite permitido según las normas, además la prueba de alcohol dio negativa y se puede afirmar que la calidad de la leche desde el punto de vista físico-químico es buena.

En análisis microbiológicos realizados por el equipo multidisciplinario de la FAREM Estelí en la investigación que se menciona anteriormente se encontró presencia de escherichia coli y salmonella en uno de los tres casos estudiados. En el análisis microbiológicos de quesos se encontró presencia escherichia coli y salmonella en uno de los cuatro casos estudiados.

Otra forma de evaluar la concentración de la leche es por medio del rendimiento. Así lo asegura el Sr. Manuel Mendoza: "lo manejo en rendimiento a la hora del proceso terminado". Los procesadores de lácteos utilizan un instrumento llamado lactodensímetro para determinar el grado de pureza que contiene la leche.
Según los procesadores, el uso de estos instrumentos les permite determinar si la leche tendrá la consistencia esperada de sus productos. A pesar de que su proceso productivo es totalmente artesanal (no utilizan maquinarias sofisticadas) sin embargo ellos logran obtener la calidad adecuada para cada uno de los productos que elaboran.

Cada uno de los procesadores de leche cuenta con una cantidad determinada de cuajo, sales y porcentaje de descremado para la elaboración de los productos.

\section{Valor Agregado}

En su mayoría, los procesadores de lácteos de la ciudad de Estelí, no cuentan con un empaque especializado para sus productos. El material que utilizan son bolsas corrientes de 1 libra y de media libra. Solamente existen procesadores (Productos Lácteos Mendoza, Productos Lácteos El Quesito y Productos Lácteos El Pastoreo) poseen un empaque más apropiado para sus productos. En estas empresas se cuenta con máquinas selladoras que permiten utilizar un material de mejor calidad para empacar sus productos.

Apenas tres empresas poseen su propia marca y etiquetan sus productos, y son: Productos Lácteos Mendoza, Productos Lácteos El Quesito y Productos Lácteos El Pastoreo.

Los procesadores consideran de gran importancia la diferenciación que se puede dar a los productos, principalmente porque permite a los consumidores distinguir un producto de otro y de esta manera no se harán acusaciones generales, sino específicas en caso de reclamo por determinado producto.

\section{Costos y utilidades}

Para abordar en detalle este punto de la investigación no se pudo recolectar la información suficiente para hacer un análisis exhaustivo de los costos 
en que incurren los procesadores de lácteos y las utilidades que les genera esta actividad comercial. A continuación se detalla un poco lo que son los costos en que se incurre en "Productos Lácteos Mendoza", datos proporcionados por el propietario de esta procesadora Señor Luís Manuel Mendoza.

\section{Costo de mano de obra y transporte:}

- Planilla semanal a C\$4,600. En promedio C\$ 575.00 por cada uno incluyendo séptimo; pero no todos tienen igual salario.

- Gasto mensual en combustible C\$ 15,000 córdobas.

\section{Gastos en otros materiales:}

- Sal: C\$2,550.00 mensual.

- Bolsas: $C \$ 3,000.00$ mensual $(C \$ 100.00$ diarios de bolsas entre bolsas de gabacha y bolsas de empaque para la crema).

- Cuajo C\$2,000.00 al mes (Dos galones de cuajo líquido marca Marshall).

La utilidad se fija a través de un promedio ya establecido; pero no es constante debido al rendimiento de la leche, ya que el grado de pureza es el que determina el rendimiento final y las cantidades producidas.

\section{El precio de comercialización}

En su totalidad, las personas a cargo de estas empresas, fijan el precio de sus productos en base a los costos. También se toma en cuenta los precios de la competencia y el rendimiento del producto para establecer los precios. Ellos determinan la totalidad de los gastos (materia prima, mano de obra, publicidad, etc.) en que se incurre a lo largo del proceso productivo y agregan a éste un margen de ganancia determinado.
Las consideraciones en cuanto a las diferencias de precios entre un productor y otro son mínimas $y$ en algunos casos son inexistentes ya que todos establecen los mismos precios. Aunque existe el caso particular de Productos Lácteos El Pastoreo, donde el señor Roberto Pérez Morales afirmaba "El precio de nuestros productos es superior a los precios establecidos por la competencia"

Los precios que se establecen son accesibles; pero existe cierta estacionalidad económica del producto debido al "Golpe de Leche". Debido al golpe de leche en invierno los precios son inferiores a los precios que se establecen durante el verano causada por la sobre producción de leche en esta época del año.

\section{La competencia}

La competencia a nivel local es casi nula ya que cada procesador tiene sus canales de distribución bien definidos; además son pocos los productores que elaboran productos novedosos como los diferentes tipos de queso y yogurt, la mayoría de los procesadores centran su proceso productivo en la elaboración de crema, cuajada y queso corriente.

Existe una competencia desleal con respecto a los productos lácteos elaborados en Chontales y Boaco ya que no hay ningún tipo de control o restricción de ingreso, no hay medidas higiénico-sanitaria para controlar estos productos y sin embargo a los productores locales se les exigen exámenes médicos del personal e inspecciones imprevistas mensuales por parte del MINSA.

\section{EI uso de la publicidad}

Las únicas dos empresas que destinan cierta cantidad de su presupuesto a la divulgación de sus productos, lo hacen a través de:

- Cuñas Radiales: utilizadas por Productos Lácteos Mendoza y Productos Lácteos El Pastoreo, estas 
son transmitidas a través de las radios con mayor audiencia a nivel local.

- Volantes: medio utilizado por Productos Lácteos Mendoza

- Publicidad de Tránsito: esta forma de hacer publicidad es utilizada por Productos Lácteos Mendoza, se realiza en taxi y camiones de su propiedad que circula por las calles de la ciudad.

Las personas que utilizan algún tipo de publicidad, hacen uso del Método de lo Factible, que consiste en destinar una cantidad de dinero determinada y muchas veces limitada, cada vez que se considera necesario divulgar los productos.

\section{La visión de desarrollo empresarial}

Los empresarios locales dedicados al procesamiento de productos lácteos tienen una buena visión en lo referente al desarrollo de su negocio y cuáles son sus perspectivas de crecimiento; es lamentable que muchos de estos deseos se vean ofuscados por la falta de apoyo hacia este sector de la economía nacional y sobre todo porque las personas que trabajan en este ramo muchas veces no se arriesgan a algo nuevo por temor.

En su mayoría los procesadores de lácteos de la Ciudad de Estelí han conservado la misma línea de productos con las que iniciaron operaciones en el mercado desde su fundación ya que no cuentan con una gama de productos diferenciados que puedan ofertar a la población esteliana y a sus mercados metas en el norte del país y algunos municipios y ciudades de occidente.

Solamente Productos Lácteos El Pastoreo y el Señor Eleazar Briones elaboran otros productos menos convencionales. Las personas entrevistadas mostraron gran interés en el hecho de elaborar nuevos productos principalmente diferentes tipos de queso, hacer innovaciones en los productos ya existentes y por supuesto ampliar su mercado porque al producir mayores cantidades, se necesitarán más compradores de estos productos.

Pretenden mejorar el proceso productivo a través de la adquisición de nuevas maquinarias (modernas) para la elaboración de los productos.

Tienen deseos de poder aumentar los niveles de producción, pero esta situación se ve afectada por el factor climático. Estas variaciones del clima es veranoinvierno, en verano disminuye la cantidad de alimento y por ende la cantidad de producción, la materia prima (leche) se torna cara en esta estación.

A pesar de la negativa que se puede observar en los procesadores de lácteos en lo referente a la presentación que le dan a los productos que elaboran, estos consideran necesario invertir en la presentación de los productos.

La mayoría de los procesadores de lácteos de esta ciudad se muestran indiferentes a lo que es la inversión en publicidad, muchos no han realizado ningún tipo de publicidad a lo largo de su existencia y tampoco pretenden hacerlo en el presente ni en el futuro.

\section{Alternativas Estratégicas de las PyMES del Sector Lácteo.}

En primer lugar es necesario determinar a qué tipo de sectores genéricos pertenecen las PyMES del sector lácteo y atendiendo las definiciones, este tipo de empresa se ubica en las industrias fragmentadas; varios factores refuerzan esta posición:

- Ninguna Empresa tiene posición de líder en el mercado, y por ende no tiene ninguna influencia en los eventos más importantes como son: los precios de los derivados de la leche y la posibilidad de consolidar un enfoque que sea imbatible para los demás competidores. 
- Existen muy bajas barreras de ingreso: Para entrar en la industria de la producción de los derivados de la leche (cuajada, crema, queso, etc), no se necesita de grandes inversiones tanto en lo que es capital fijo, como capital de trabajo. Además de lo anteriormente expuesto el nivel de desarrollo tecnológico es muy bajo.

- Ausencia de economías de escala: Difícilmente en las industria que procesan los derivados de la leche, se puede observar que éstas practique las economías de escala, debido a que los volúmenes de producción son muy bajos.

- Producto estándar: Casi no se observa diferencias sustanciales en la producción de cada una de las PyMES que intervienen con sus productos en el mercado de derivados de la leche.

Lo antes expuesto demuestra la existencia de una gran cantidad de competidores y una débil capacidad de negociación con los proveedores de leche, por ello su nivel de rentabilidad es muy modesto en relación a otras industrias del contexto nacional.

Para cualquier PyMES que interviene en esta coyuntura es un enorme desafío consolidarse como una empresa que reporte utilidades por encima de la media y le permita crecer en este medio. Existen algunas alternativas estratégicas a considerar, que pueden contribuir a que estas empresas mejoren sus niveles de competitividad, a continuación analizamos cada una de ellas:

- Valor Agregado Incrementado: Significa proporcionar productos que incluya dentro del mismo algún tipo de diferenciación, esto sobre todo es válido en lo que respecta a los servicios ex - venta; como post- venta. Por ejemplo estas empresas podrían asegurar un incremento en las ventas si están en la posibilidad de llevar hasta los clientes que compran grandes volúmenes su producción, agregándole a esto descuento sobre el producto.
- Especialización Por Tipo de Producto: Si la fragmentación obedece a la presencia de una gran cantidad de productos en el mercado, una manera de consolidar una posición muy difícil de batir por parte de los competidores es la especialización en una reducida gama de productos en los cuales posea ventajas competitivas; por ejemplo la especialización trae como consecuencia un aumento en la productividad del trabajo. Para esta alternativa estratégica es importante analizar los costos de cada uno de los productos y determinar cuáles son aquellos que ofrecen una mayor posibilidad de aumento en la rentabilidad de la empresa.

- Enfocarse en un Área Estratégica: Segmentar el mercado, esta manera puede traer como beneficio el conocer muy bien las necesidades de los consumidores de este mercado geográfico y por ende satisfacer las mismas. Este tipo de estrategia exige que periódicamente la empresa realice estudios de mercado para determinar cuáles son las nuevas necesidades que pueden surgir en el segmento geográfico que domina. Es perentorio tener en cuenta que las necesidades de los consumidores son cada día más cambiantes.

- Franqueza sin presunciones: Ante la imposibilidad que presentan las PyMES del sector lácteo de generar economías de escala es imprescindible el estricto control de los costos a los niveles mínimos posibles. La austeridad debe ser uno de los valores empresariales principales que deben observar este tipo de empresas.

- Diferenciación de la Imagen de los Productos: La diferenciación de la imagen se puede alcanzar a través del aprovechamiento de empaques vistosos que refuercen el posicionamiento de la marca de la empresa en la mente de los consumidores. La consolidación de la marca se logra cuando realmente el producto tiene ciertos atributos que le son difíciles a la competencia de igualar o superar.

- Integración en Sentido Inverso o Directo: En este sentido muchas pequeñas empresas del 
sector lácteo podrán abaratar sus costos, si en vez de comprar la materia prima (la leche); sean ellos mismo las que la producen de esta manera tendrían una integración en sentido inverso o bien a través de la integración directa, cuando la empresa utilice sus propios canales para llegar hasta el consumidor final.

\section{CONCLUSIONES}

La pequeña industria láctea que existe en la ciudad de Estelí se enfrenta a muchas dificultades internas y externas. Cuando se habla de dificultades externas se hace referencia a aquellas dificultades que son provocadas por las normativas y reglamentos que se dictan para regular la actividad de estas pequeñas empresas a nivel local.

Las autoridades locales exigen a los procesadores locales exámenes médicos del personal e inspecciones imprevistas mensuales por parte del MINSA; sin embargo permiten la distribución de productos lácteos elaborados en Chontales y Boaco sin ningún tipo de control o restricción de ingreso, no hay medidas higiénico-sanitaria para controlar estos productos y esto es un grave error ya que los productos provenientes de estos departamentos son nocivos para la salud por que contienen formalina para evitar la descomposición de la materia prima.

Los problemas que presentan las empresas a nivel interno son los siguientes:

- La materia prima (leche), que se utilizada en el proceso productivo, muchas veces no presenta la consistencia necesaria debido a alteraciones realizadas en el punto de ordeño.

- A pesar del planteamiento que hacen los procesadores de lácteos acerca de la importancia de diferenciar sus productos, es evidente que este punto no les interesa mucho ya que no muestran preocupación por dar una buena imagen a sus productos, la mayoría de ellos no tienen una marca, no etiquetan sus productos y el empaque es bastante tradicional.

- No cuentan con un plan de publicidad, divulgan sus productos cuando lo creen sumamente necesario.

En resumen, la hipótesis planteada se cumple parcialmente. Se demostró que para las empresas procesadoras de lácteos de la ciudad de Estelí no representan algún tipo de problema los precios que fijan a sus productos, además los productos que se elaboran son de calidad. El problema de la publicidad y la limitada distribución de los productos está influenciada por la visión que tiene cada empresario, en general estos no hacen uso de los medios publicitarios que les ofrece el mercado y que son de gran ayuda para dar a conocer sus productos y de esta forma poder hacer crecer su negocio y sus canales de distribución son limitados debido a que aún no se arriesgan a ir más allá de las fronteras que tienen fijadas en la actualidad.

\section{BIBLIOGRAFIA}

J. M. Rosenberg (1997). Diccionario de Administración y Finanzas. OCEANO GRUPO EDITORIAL, S. A. Barcelona, España.

Kotler, Philip y Armstrong, Gary (2001). Marketing. Octava Edición. PEARSON ADUCACIÓN. México. Kotler, Philip; Armstrong, Gary; Cámara Ibáñez, Dionisio y Cruz Roche, Ignacio (2004). MARKETING. Décima Edición. PEARSON EDUCACIÓN, S.A. Madrid, España.

Piura López, Julio (2000). Introducción a la metodología de la investigación científica. Centro de investigación y estudios de la salud (CIES). Cuarta Edición. Managua, Nicaragua.

Aguilar, Omar y Morales Omar (2003). COMERCIALIZACIÓN DE PRODUCTOS AGROPECUARIOS; Material de estudio para el fortalecimiento de las organizaciones beneficiarias del programa PAC-UESA. Managua, Nicaragua. 
Gaitán, Fabio José (2004). Folletos de la clase Mercado II. FAREM. Estelí, Nicaragua.

Gaitán, Fabio José (2005). Folletos de la clase Mercadotecnia Internacional. FAREM. Estelí, Nicaragua.

Navarro Zeledón, Mauricio (2006). Folletos de la clase Publicidad. FAREM. Estelí, Nicaragua.

Rodríguez Pérez, Máximo (2007). Folletos de la clase Formulación y Evaluación de Proyectos. FAREM. Estelí, Nicaragua.

Navarro Zeledón, Mauricio (2007). Folletos de la clase Estrategia Empresarial. FAREM. Estelí, Nicaragua. Herrera, E., Navarro, M. (2007). "Diagnóstico de la Calidad y comercialización de Leche y sus Derivados en el Municipio de Estelí" FAREMEstelí, Nicaragua.

Entrevista a Eleazar Briones realizada por Arlen Picado Juárez el día domingo 6 de Mayo de 2007.

Entrevistas a productores de lácteos del municipio de Estelí(noviembre 2007):

Sr. Fernando Hidalgo.

Sr. Luis Manuel Mendoza (Productos Lácteos Mendoza).

Sr. Julio Cesar Galiano (Productos Lácteos Mendoza).

Sr. Eleazar Antonio Briones Valle.

Lic. Roberto Pérez Morales (Productos Lácteos El Pastoreo).

Sra. Luz Mery Molina Moreno (Productos Lácteos René Valle).

Sra. Carmen Vílchez Castillo (Productos Lácteos El Quesito).

Sra. Miriam Antonia Rugama

Leonardo Pérez (Productos Lácteos El Pastoreo).

Entrevista al Dr. Bismark Rodríguez Montenegro.

Centro de Salud Leonel Rugama-Estelí realizada el día 21 de Noviembre de 2007.

ProNicaragua Investment Promotion Agency Potencial ganadero de Nicaragua es muy alto.htm Ervin Filemón Sánchez "Ganadería nacional con buen futuro". Sitio web: http//impreso.elnuevodiario. com.ni/lunes20agosto2007.Edición9705.

Comentarios de lectores Autor: Gustavo Álvarez,
El Nuevo Diario. Producción lechera aumenta rápidamente el "La Vía Láctea". Jueves 29 de junio 2006. Sitio web: www.guegue.com 